\title{
Suppression of transcription factor early growth response 1 reduces herpes simplex virus lethality in mice
}

\author{
Shih-Heng Chen, ${ }^{1}$ Hui-Wen Yao, ${ }^{1}$ I-Te Chen, ${ }^{2}$ Biehuoy Shieh, ${ }^{3}$ Ching Li, ${ }^{4}$ and Shun-Hua Chen ${ }^{1,2}$ \\ ${ }^{1}$ Institute of Basic Medical Sciences and 2Department of Microbiology and Immunology, College of Medicine, National Cheng Kung University, Tainan, \\ Republic of China. ${ }^{3}$ Department of Pharmaceutical Science, University of Colorado at Denver and Health Sciences Center, Denver, Colorado, USA. \\ ${ }^{4}$ Department of Microbiology and Immunology, College of Life Sciences, National Chiayi University, Chiayi, Republic of China.
}

\begin{abstract}
Herpes simplex virus type 1 (HSV-1) infection is the most common cause of sporadic, fatal encephalitis, but current understanding of how the virus interacts with cellular factors to regulate disease progression is limited. Here, we show that HSV-1 infection induced the expression of the cellular transcription factor early growth response 1 (Egr-1) in a human neuronal cell line. Egr-1 increased viral replication by activating promoters of viral productive cycle genes through binding to its corresponding sequences in the viral promoters. Mouse studies confirmed that Egr-1 expression was enhanced in HSV-1-infected brains and that Egr-1 functions to promote viral replication in embryonic fibroblasts. Furthermore, Egr-1 deficiency or knockdown of Egr-1 by a DNA-based enzyme greatly reduced the mortality of HSV-1-infected mice by decreasing viral loads in tissues. This study provides what we believe is the first evidence that Egr-1 increases the mortality of HSV-1 encephalitis by enhancing viral replication. Moreover, blocking this cellular machinery exploited by the virus could prevent host mortality.
\end{abstract}

\section{Introduction}

Herpes simplex virus type 1 (HSV-1) infects about $80 \%$ of young adults worldwide and induces encephalitis (1-3). HSV-1 encephalitis is the most devastating consequence of all HSV infections and also the most common cause of sporadic, fatal encephalitis, with an incidence of 1 in 200,000 individuals per year (1). The mortality rate of untreated patients is over $70 \%$, and only $2.5 \%$ of all patients return to normal neurological function (1). Once the virus enters cells, it interacts with cellular factors to increase viral replication and mortality in infected hosts. Identifying these cellular factors is essential for gaining a better understanding of HSV-1 pathogenesis and should provide cellular targets for developing alternative strategies to prevent host mortality. However, little is known about these cellular factors and their mechanisms.

Early growth response 1 (Egr-1), also known as NGFI-A, Zif268, TIS-8, Z225, and Krox-24, is a zinc finger transcription factor constitutively expressed, particularly in neural tissues (4-7), and can also be induced upon stress (8-12). It is shown to regulate many cellular activities, such as growth, proliferation, apoptosis, angiogenesis, and development $(6,7)$, but its deficiency does not result in obvious defects except that female mice lacking Egr-1 are infertile (5, 7, 13). Several viruses and viral proteins have been shown to induce Egr-1 expression $(9-12,14)$, and Egr-1 is known to regulate several viral genes (14-16), including the gene-encoding latency-associated transcripts (LATs) of HSV-1 (17). However, the functions of Egr-1 in viral replication and disease progression remain unclear. In the search for cellular factors interacting with HSV-1 to regulate viral infection, we found that transcription factor Egr-1 was induced after

Nonstandard abbreviations used: DNAzyme, DNA-based enzyme; Egr-1, early growth response 1 ; HSV-1, herpes simplex virus type 1; LAT, latency-associated transcript; p.i., post infection.

Conflict of interest: The authors have declared that no conflict of interest exists. Citation for this article: J. Clin. Invest. 118:3470-3477 (2008). doi:10.1172/JCI35114. infection. Our results showed that Egr-1 increased viral replication in infected cells and mouse tissues and mortality in infected mice. Furthermore, knockdown of Egr-1 expression reduced the mortality of infected mice by decreasing viral loads in tissues.

\section{Results}

HSV-1 infection induces Egr-1 expression in both human cells and mouse tissue. To search for cellular factors regulating HSV-1 replication, we infected a human neuronal cell line, SK-N-SH, with HSV-1 strain KOS and harvested cultures to investigate viral replication and changes in cellular gene expression. The viral titers of infected cultures increased between 12 and 48 hours post infection (p.i.) and reached $10^{7}$ PFUs per culture at 48 hours p.i. (Figure 1A). We used microarray to analyze total RNA isolated from infected cells from 0 to 48 hours p.i. to identify cellular factors whose gene expression is altered during infection because such factors could be candidates for controlling viral infection. Microarray results showed that the expression of several cellular genes was altered during infection (Supplemental Figure 1; supplemental material available online with this article; doi:10.1172/ JCI35114DS1). We performed studies and found that knockdown of Egr-1 affected viral growth in SK-N-SH cells. Therefore, we further characterized Egr-1 induction during infection. RT-PCR analysis showed that the expression of Egr-1 mRNA in infected SK-N-SH cells gradually increased from 2 through 48 hours p.i. (Figure 1B), which is consistent with the microarray results (Supplemental Figure 1). Western blot analysis showed that Egr-1 protein was induced in SK$\mathrm{N}$-SH cells infected with infectious virus but not with UV-inactivated virus (Figure 1C). Additional results using reporter assay and Egr-1 promoters with various lengths of deletion showed that HSV-1 infection activated Egr-1 promoter in SK-N-SH cells via interaction with the $5^{\prime}$ end of the promoter (Supplemental Figure 2).

We next studied Egr-1 induction in vivo using Egr-1 wild-type $\left(E g r-1^{+/+}\right)$and deficient $\left(E g r-1^{-/-}\right)$C57BL/6 mice $(5,7,13)$ infected with HSV-1 strain 294.1 (18) via corneal inoculation. Our pre- 
A

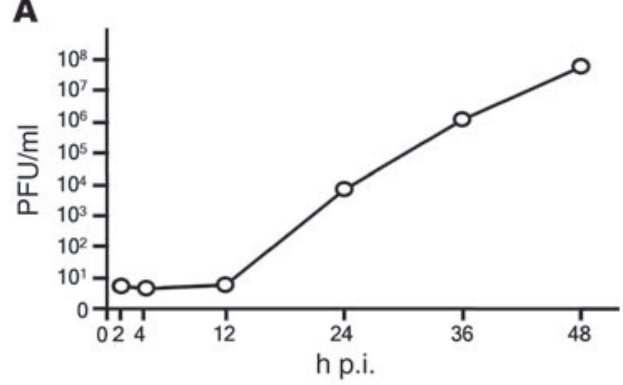

B

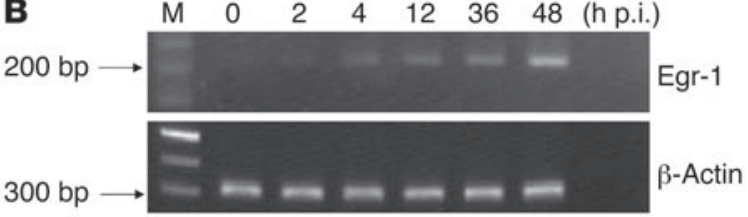

C
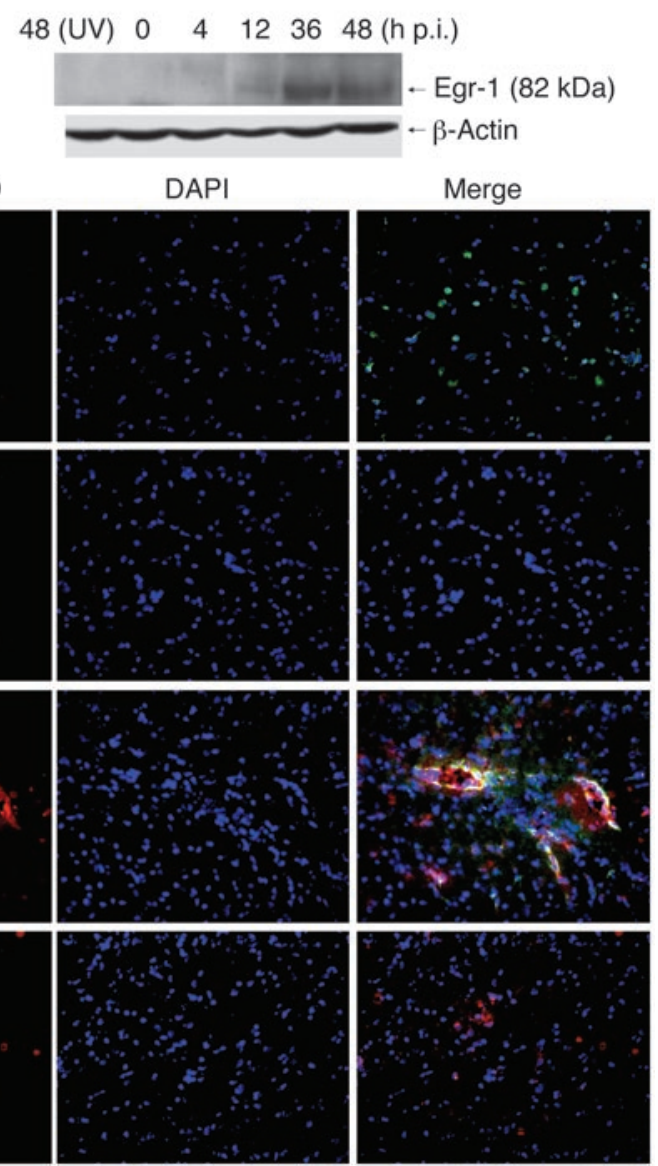

Figure 1

HSV-1 infection increases Egr-1 expression. The viral growth (A) and Egr-1 expression assayed by RT-PCR (B) and Western blot (C) analyses were monitored in SK-N-SH cells infected with strain KOS or with UV-inactivated virus (UV). M, molecular weight marker. (D) The brain stem

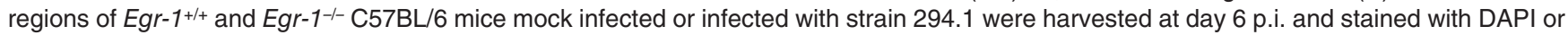
antibodies against Egr-1 or viral antigen glycoprotein D (gD). Original magnification, $\times 200$. Data are representative of at least 2 experiments.

vious study showed that the majority of virus in the brains of HSV-1-infected mice was found in the brain stem region (19). Accordingly, mouse brains were harvested at day 6 p.i. to detect the expression of Egr-1 and viral antigen (glycoprotein D) in the brain stem region using immunofluorescence staining. Egr-1 expression was not detected in the brains of either mock-infected or infected Egr-1 $1^{-/-}$mice (Figure 1D). Egr-1 was expressed at basal levels in the brains of mock-infected $E g r-1^{+/+}$mice. In the brains of infected $E g r-1^{+/+}$mice, Egr-1 expression was enhanced mostly in cells where viral antigen was detected. Collectively, these results demonstrate that HSV-1 infection induces Egr-1 expression in both human cells and mouse tissue.

Egr-1 functions to increase HSV-1 replication in both buman and mouse cells. To determine the function of Egr-1 in HSV-1 replication, we knocked down Egr-1 protein expression in SK-N-SH cells using an antisense oligomer previously shown to effectively reduce Egr-1 protein expression to a basal level $(20,21)$. The resulting cells were infected with strain KOS and then harvested for titration. The mean viral titers in the SK-N-SH cells treated with the Egr-1 antisense oligomer were lower than those treated with a control (scramble) oligomer from 12 through 48 hours p.i., with a significant (10-fold) difference at 36 hours p.i. (Figure 2A; $P=0.01$, Student's $t$ test).

In addition, we cultured mouse embryonic fibroblasts from $E g r-1^{+/+}$and $E g r-1^{-/-}$mice, infected the cells with strain KOS, and harvested infected cultures for titration. The mean viral titers in the embryonic fibroblasts from $\mathrm{Egr}-\mathrm{1}^{-/-}$mice were lower than those from $E g r-1^{+/+}$mice from 12 through 48 hours p.i., with a significant (7-fold) difference at 24 hours p.i. (Figure 2B; $P=0.03$, Student's $t$ test). We also examined the growth of strain KOS in the brain slices prepared from $E g r-1^{+/+}$and $E g r-1^{-/-}$mice. Figure $2 \mathrm{C}$ shows that the mean viral titer in the brain slices from $E g r-1^{-/-}$mice was significantly lower than that from $E g r-1^{+/+}$mice, with 5-fold differ- 

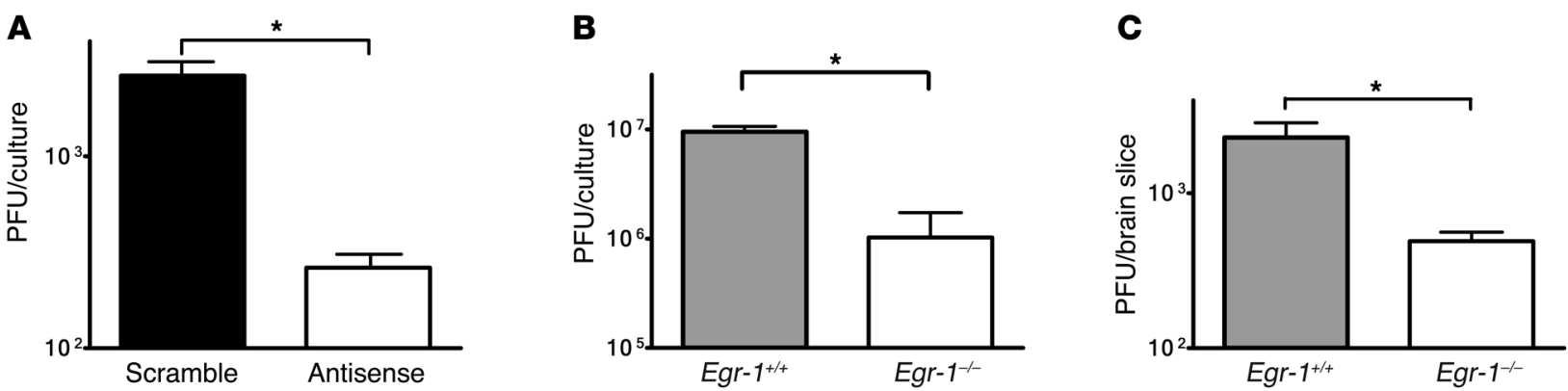

Figure 2

Egr-1 increases HSV-1 replication. Growth of strain KOS in SK-N-SH cells transfected with an antisense or scramble oligomer to Egr-1 (A) or in embryonic fibroblasts (B) and brain slices (C) prepared from Egr-1+/+ or Egr-1-/- mice. Data show mean \pm SEM from 2 independent experiments, each done in duplicate. ${ }^{*} P<0.05$ (Student's $t$ test), relative to antisense oligomer or Egr-1/- mice.

ence at 24 hours p.i. ( $P=0.03$, Student's $t$ test $)$. Collectively, these results demonstrate that the Egr-1-mediated increase of HSV-1 replication is not a phenomenon specific to human or mouse cells or to a particular cell type.

Egr-1 activates the productive cycle gene promoters of HSV-1 by binding to its corresponding sequences in the promoters. Egr-1 is a transcription factor shown to activate the promoters of immediate-early genes important for productive infection $(14,15)$ and to suppress the promoter of the latent gene (16) of Epstein-Barr virus. With regard to HSV-1, Egr-1 is shown to repress the promoter of the latent gene encoding LATs (17). If Egr-1 increases HSV-1 replication in cells through repressing LAT, the replication of LAT deletion mutants in cells should be increased compared with that of wild-type viruses. However, our results found that the replication of 2 LAT deletion mutants, $d$ LAT2903 (22) and dlLAT1.8 (23), and their wild-type viruses, McKrae and KOS, was comparable in SK-N-SH cells (data not shown), which is consistent with previous studies using other cell lines $(22,23)$. These results indicate that Egr-1 functions to increase HSV-1 replication in cells in a LAT-independent manner.

We next investigated whether Egr-1 increases HSV-1 replication in infected cells by activating the expression of viral productive cycle genes as it does for Epstein-Barr virus $(14,15)$. We performed a search of Egr-1-binding sequences in the genome of HSV-1 strain 17 (GenBank accession no. NC001806) and found at least 7 viral gene promoters containing Egr-1-binding sequences (24-28), the promoters of 4 immediate-early genes (ICP0, 27, and ICP22/47), 1 early gene (ICP6), 1 late gene (ICP5), and LAT (17, 29-32). We also found an Egr-1-binding sequence in the promoter of immediateearly gene ICP4 when we cloned it from HSV-1 strain RE (GenBank accession no. EF667506). To further study whether Egr-1 activates HSV-1 immediate-early gene promoters containing Egr-1-binding sequences, we established clones of SK-N-SH cells stably expressing
A

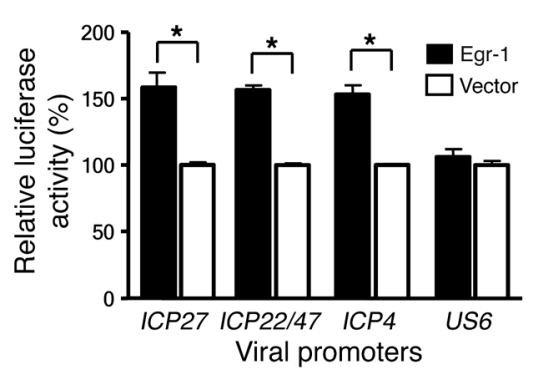

B

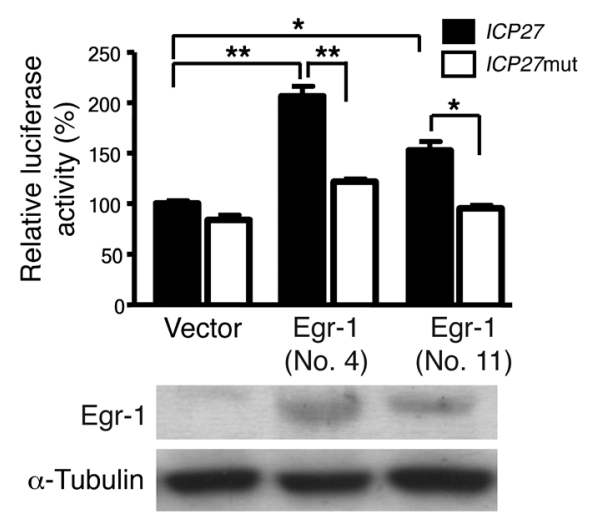

C

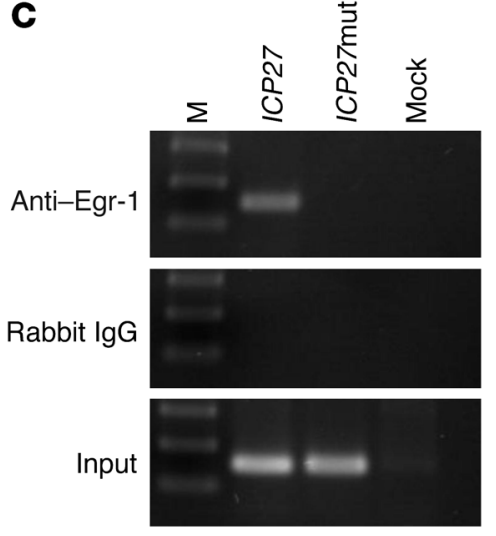

Figure 3

Egr-1 activates HSV-1 gene promoters by binding to its corresponding sequence in the promoter. (A) The activities of the ICP27, ICP22/47, ICP4, and US6 promoters in SK-N-SH cells stably transfected with plasmids containing Egr-1 (Egr-1) or empty vector (vector). (B) The activities of the ICP27 promoter (ICP27) or ICP27 promoter with a mutated Egr-1-binding sequence (ICP27 mut) (top panel) and Western blot analysis of Egr-1 (middle panel) and $\alpha$-tubulin (bottom panel) in 1 clone of SK-N-SH cells stably transfected with vector plasmid and 2 clones of SK-N-SH cells stably transfected with Egr-1 plasmid, clone numbers 4 (Egr-1 no. 4) and 11 (Egr-1 no. 11). The viral promoter activities in cells stably transfected with $\mathrm{pCB} 6$ were set at $100 \%$. Data show mean \pm SEM from 3 independent experiments, each done in duplicate. ${ }^{*} P<0.05$; ${ }^{* *} P<0.005$, Student's $t$ test. (C) Binding of Egr-1 to its corresponding sequence in the ICP27 promoter assayed by ChIP-PCR assay. Egr- 1 and DNA complex from cells mock transfected (mock) or transfected with plasmids containing the ICP27 (ICP27) or mutated ICP27 (ICP27 mut) promoter was precipitated by anti-Egr-1 antibody or control rabbit IgG. The precipitated DNA and genomic DNA (input) were subjected to PCR amplification using primers specific for both ICP27 and mutated ICP27 promoters. Data are representative of at least 2 experiments. 
Table 1

Viral loads in the tissues of HSV-1-infected Egr-1 mice

$\begin{array}{ccccc}\text { Mouse } & & \text { Day 5 p.i. } & & \text { Day 7 p.i. } \\ & \text { Eye } & \text { Trigeminal ganglion } & \text { Brain } & \text { Trigeminal ganglion } \\ \text { Egr-1+/+ } & 4.0 \pm 0.2(n=6) & 3.6 \pm 0.1(n=8) & 3.1 \pm 0.2(n=6) & 1.1 \pm 0.2(n=11) \\ \text { Egr-1+- } & 4.3 \pm 0.3(n=7) & 3.8 \pm 0.1(n=8) & 3.4 \pm 0.3(n=7) & 0.7 \pm 0.3(n=6) \\ \text { Egr-1-/ } & 4.5 \pm 0.4(n=8) & 3.4 \pm 0.1(n=8) & 3.3 \pm 0.4(n=8) & 0.7 \pm 0.3(n=8)\end{array}$

Mouse tissues were harvested at indicated times after infection to titrate for infectious virus. All data show titer in tissues $\left(\log _{10}\right.$ mean PFU per tissue \pm SEM).

Egr-1 and then transfected the stable cell clones with plasmids containing the promoters of viral immediate-early genes ICP4, ICP27, or ICP22/47, all linked to a luciferase reporter gene. The promoter of a late gene (US6) that does not contain any Egr-1-binding sequence, was used as a negative control. Egr-1 significantly increased the activities of ICP4, ICP27, and ICP22/47 promoters, which contain Egr-1-binding sequences $(P<0.05$, Student's $t$ test), but failed to alter the activity of US6 promoter (Figure 3A). Among the stable cell clones expressing Egr-1, clone 4 was shown by Western blot analysis to express a higher level of Egr-1 than clone 11 (Figure 3B). Notably, the ICP27 promoter activity of clone 4 was higher than that of clone 11 (Figure 3B), indicating that the viral promoter activities are correlated with the levels of Egr-1 expressed in cells. To further identify whether Egr-1 activates the viral promoter by binding to its corresponding sequence, we mutated the Egr-1-binding sequence in the promoter of an essential gene, ICP27, from $5^{\prime}$-GCGGGGGCC-3' to $5^{\prime}$-TAAGCTTTA- $3^{\prime}$ and determined the promoter activity. The activity of the ICP27 promoter was decreased when the Egr-1-binding sequence was mutated (Figure 3B). Subsequently, we determined whether Egr- 1 indeed binds to its predicted binding sequence in the ICP27 promoter 48 hours after transfection, using ChIP-PCR assay. Our results showed that Egr-1 bound to its binding site in the ICP27 promoter and that the binding was abolished when the binding sequence was mutated (Figure 3C). Collectively, these results suggest that Egr-1 upregulates viral promoter activities via binding to its corresponding sequences in the promoters.

Egr-1 increases the viral loads in tissues and mortality of infected mice. We next examined the functions of Egr-1 in HSV-1 infection in vivo using $E g r-1^{+/+}, E g r-1^{+/}$, and $E g r-1^{-/-}$mice infected with strain 294.1. The viral growth in tissues and mortality of infected mice were monitored. The mean viral titers in the eyes, trigeminal ganglia, and brains at day 5 p.i. and in the trigeminal ganglia at day 7 p.i. of $\mathrm{Egr}_{-1^{+/+}}, \mathrm{Egr-1} \mathrm{1}^{+-}$, and $\mathrm{Egr-1} \mathrm{1}^{-/-}$mice were comparable (Table 1). At day 7 p.i., the mean viral titer in the eyes of $\mathrm{Egr}^{-1^{+/}}$mice was 4- and 155-fold higher than that of Egr-1 $1^{+/-}$and Egr-1-/- mice and the mean viral titer in the brains of $E g r-1^{+/+}$mice was 3- and 10-fold higher than that of $E g r-1^{+/-}$and $E g r-1^{-/-}$mice, respectively

\section{Figure 4}

Egr-1 increases the viral loads in tissues and mortality of HSV-1infected mice. (A) The viral loads in the eyes and brains of Egr-1+/+ $(n=11)$, Egr-1+/- $(n=6)$, and Egr-1/-/ $(n=8)$ mice infected with strain 294.1 at day 7 p.i. Data show mean \pm SEM. ${ }^{*} P=0.005$, Mann-Whitney $U$ test. (B) Deaths of Egr-1+/+ $(n=32), E g r-1^{+/-}(n=11)$, and Egr-1-1- $(n=12)$ mice infected with strain 294.1. ${ }^{\dagger} P=0.009$, log-rank test. (C) Deaths of Egr-1+/+ $(n=20)$ and Egr-1-1- $(n=6)$ mice infected with strain $d$ LAT2903.
(Figure 4A). By day 40 p.i., the mortality of infected $\mathrm{Egr}_{\mathrm{-1}} \mathrm{1}^{+/}, \mathrm{Egr-1^{+/ }}$, and Egr-1 $1^{-/-}$mice was $44 \%, 18 \%$, and $0 \%$, respectively (Figure 4B). The viral titer in mouse eyes at day 7 p.i. and mortality of $E g r-1^{+/+}$mice were significantly higher than in $E g r-1^{-/-}$mice $(P=0.005$, Mann-Whitney $U$ test, and $P=0.009, \log$-rank test, respectively). During acute infection, $E g r-1^{+/+}$mice showed more severe signs of encephalitis, manifested by hunched posture, lethargy, ataxia, and anorexia. At day 9 p.i., more damaged neurons and inflammatory infiltrates were observed in the brain stems of $\mathrm{Egr-1}^{+/+}$mice when compared with Egr-1-1/- mice (Figure 5). In some areas of the brain stems of Egr-1//+ and $E g r-1^{-/-}$mice, approximately $25 \%$ and less than $10 \%$ of neurons were found to be damaged (Figure 5, G and H), and approximately 37 and 11 mononuclear cells were found (Figure 5, E and F), respectively. Collectively, these results demonstrate that Egr-1 increases the mortality of HSV-1-infected mice by enhancing viral replication and neuron damage in brains.

We also used dLAT2903 (22), a LAT-deletion mutant derived from HSV-1 strain McKrae, to infect mice (Figure 4C). This virus still caused mortality in $\mathrm{Egr-1^{+/+ }}$ mice (40\%) but not in $\mathrm{Egr-1^{-/- }}$ mice $(0 \%)$ in a trend similar to that seen when strain 294.1 was used for infection (Figure 4B). These data indicate that the Egr-1-mediated increase in the mortality of HSV-1-infected mice is not a phenomenon specific to a particular viral strain.

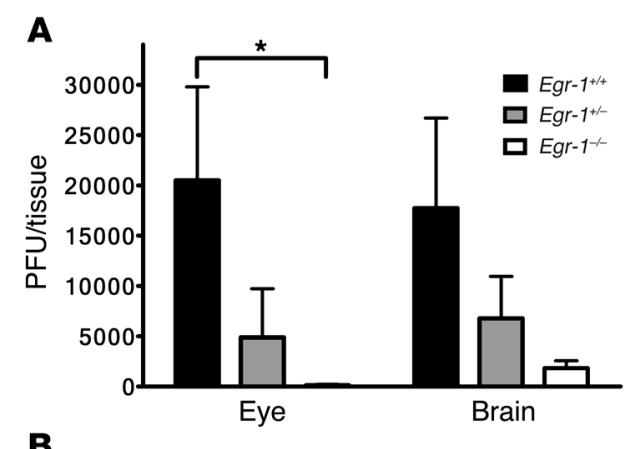

B

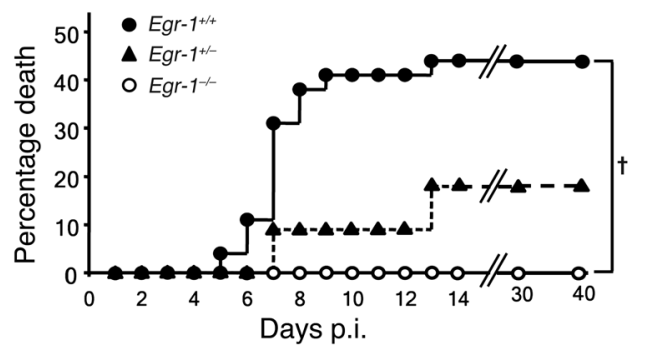

C

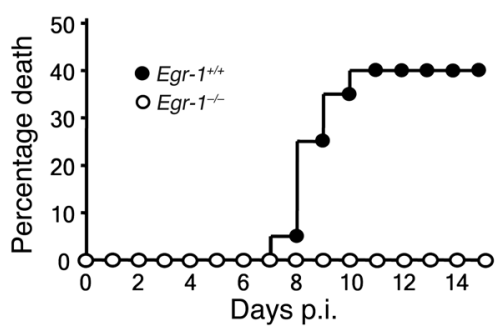



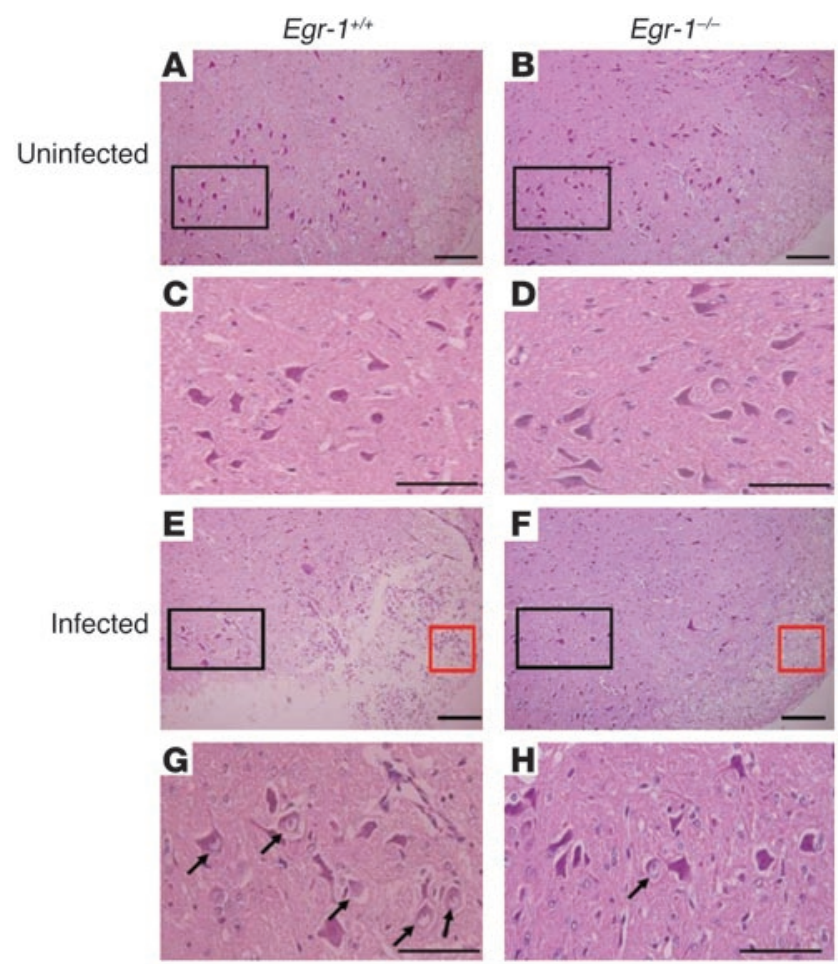

Knockdown of Egr-1 expression by a DNA-based enzyme reduces the viral loads in tissues and mortality of infected mice. Egr-1 knockdown by DNAbased enzymes (DNAzymes) has been shown to successfully reduce angiogenesis and tumor growth in mice $(33,34)$. Therefore, we investigated whether Egr-1 knockdown would decrease the mortality of HSV-1 encephalitis in mice by reducing viral loads in tissues. Wildtype C57BL/6 mice were treated with a DNAzyme (ED5) against Egr-1 that has been shown to effectively reduce Egr-1 protein expression $(33,34)$, or a scramble oligomer (ED5SCR) topically on the inoculation site (cornea) and systemically by intravenous injection right before infection with strain 294.1. Figure 6A shows that ED5 significantly reduced the mean viral titer in mouse brains by 11 -fold ( $P=0.026$, Mann-Whitney $U$ test) when compared with ED5SCR. ED5 also reduced the mean viral titers in mouse eyes and trigeminal ganglia by 35- and 3.3-fold. Furthermore, the mortality of infected mice treated with ED5 (0\%; Figure 6B) was significantly lower than that of mice treated with ED5SCR (50\%; $P=0.05$, log-rank test). We also tested mice given only topical treatment and found that ED5 reduced the mean viral titer in mouse brains by approximately 2 -fold but failed to reduce viral replication in mouse trigeminal ganglia and eyes when compared with ED5SCR (Supplemental Figure 3). Thus, topical plus systemic treatment was more effective than topical treatment in suppressing viral replication in mouse tissues.

\section{Figure 6}

Knockdown of Egr-1 reduces the viral loads in tissues and mortality of HSV-1-infected mice. (A) The viral loads in the brains, trigeminal ganglia, and eyes of wild-type C57BL/6 mice treated with a DNAzyme blocking Egr-1 expression (Egr-1 DNAzyme, $n=6$ ) or a scramble oligomer (scramble, $n=6$ ) and infected with strain 294.1 at day 5 p.i. Data show mean \pm SEM. ${ }^{*} P=0.026$, Mann-Whitney $U$ test. (B) Deaths of infected mice treated with the Egr-1 DNAzyme $(n=6)$ or the scramble oligomer $(n=6)$. ${ }^{+} P=0.05$, log-rank test.

\section{Figure 5}

Inflammatory cell infiltration and neuron damage in infected mouse brain stems. Sections of brain stems from uninfected (A-D) and infected $E g r-1^{+/+}$and $E g r-1^{-/-}$mice $(E-H)$ stained with $\mathrm{H} \& \mathrm{E}$ are shown. The black boxes in A, B, E, and F are enlarged and shown in C, D, G, and $\mathbf{H}$, respectively. More inflammatory cellular infiltration (red boxes in $\mathbf{E}$ and $\mathbf{F}$ ) and damaged neurons with swollen nuclei (arrows in $\mathbf{G}$ and H) are noted in the brains of infected Egr-1/++ mice at day 9 p.i. Scale bars: $100 \mu \mathrm{m}$. Data are representative of 2 experiments.

\section{Discussion}

The present study provides what we believe is the first evidence that Egr-1 acts as an enhancer for HSV-1 replication by binding to and activating the promoters of viral genes important for productive infection. Furthermore, suppression of Egr-1 reduces the viral replication in infected cells, viral loads in mouse tissues, and mortality in infected mice with HSV-1 encephalitis. This study demonstrates that HSV-1 hijacks cellular factors to increase viral replication and disease progression in infected hosts; the study also enhances our understanding of HSV-1 pathogenesis. Besides HSV-1, several other viruses, such as rabies virus and Borna disease virus, and viral proteins of Epstein-Barr virus and human $\mathrm{T}$ cell leukemia virus have been shown to induce Egr-1 $(9-12,14)$. Furthermore, Egr-1 is shown to regulate the gene promoters of Epstein-Barr virus and HSV-1 in a similar way (14-17). However, the roles of Egr-1 in these viral infections remain to be addressed.

The Zta protein of Epstein-Barr virus has been shown to activate the Egr-1 promoter (14). Our in vitro results showed that infectious but not UV-inactivated HSV-1 induces Egr-1 expression, indicating that the viral replication process is required for induction. Our additional results found that HSV-1 infection activates Egr-1 promoter activity in SK-N-SH cells via interaction with the $5^{\prime}$ end of the promoter (Supplemental Figure 2), which contains a cAMP response element and binding sites for several transcription factors, including SP1, AP1, and Egr-1 itself (35). HSV infection has been shown to increase the expression or modify the activities of cAMP, SP1, and AP1 (36-38), and the present study shows that HSV-1 infection
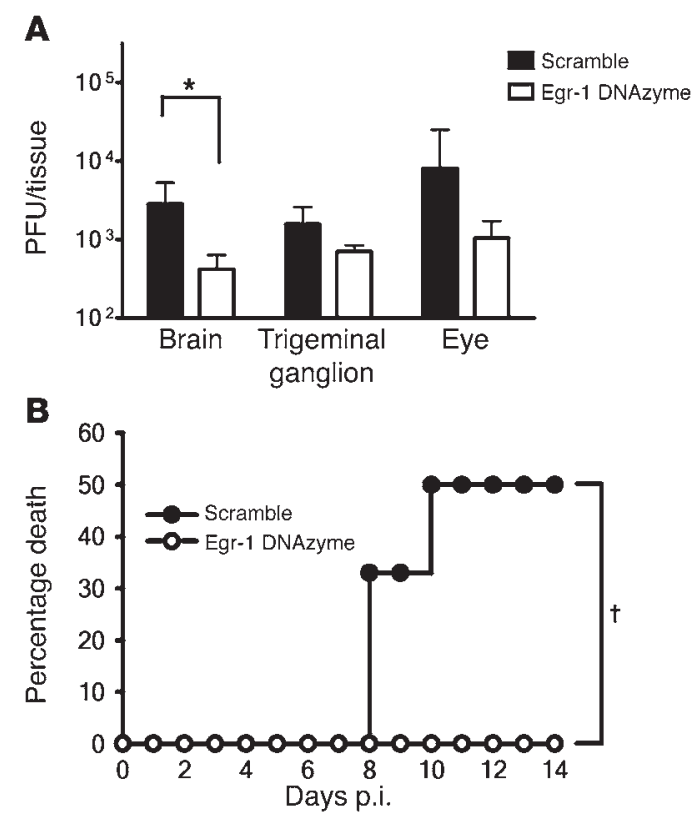
induces Egr-1. Whether these activators, HSV-1 protein(s), or other factors induce Egr-1 expression during infection is a question that remains to be investigated. Additionally, our in vivo results found that few viral antigen-negative cells near antigen-positive, infected cells expressed Egr-1 in the brains of infected mice, indicating that some uninfected cells may express Egr-1 as a response to the factors or paracrine effects induced by HSV-1 infection.

In this study, Egr-1 expression promoted viral persistence, especially in mouse eyes. However, this did not increase the incidence of virus-induced corneal blindness (stromal keratitis), probably because the viral strains (294.1 and $d$ LAT2903) used to infect mice are not proficient inducers of eye disease (data not shown). After acute infection, HSV-1 establishes latency in mouse trigeminal ganglia. We found that all the trigeminal ganglia harvested from both $E g r-1^{+/+}$and $E g r-1^{-/-}$mice infected with strain 294.1 at day 30 p.i. reactivated virus (data not shown), demonstrating that Egr1 did not affect HSV-1 latency in the mouse trigeminal ganglia under our experimental conditions.

In infected mice, a gene dosage effect of Egr-1 in increasing the viral loads in tissues and mortality of infected mice was observed. In the future, it will be of interest to investigate the influence of Egr-1 expression on human susceptibility to HSV-1 encephalitis. In humans, individuals with immunodeficiencies are shown to be very susceptible to HSV-1 encephalitis $(3,39)$ and to need antiherpetic therapies. Due to the severity of HSV-1 encephalitis, more antiherpetic therapies are needed. In addition, all antiherpetic drugs currently available are directed against viral proteins (1). However, their extensive clinical use has led to the emergence of resistant viral strains due to virus mutations. There is a need to develop alternative therapies for the treatment of drug-resistant viral strains. Our study showing that knockdown of Egr-1 prevents the mortality of HSV-1-infected mice by reducing viral replication in tissues provides a cellular target for developing alternative therapies. Further studies are needed to test whether blocking Egr-1, which is unlikely to mutate, could be an alternative therapy for preventing HSV-induced diseases or reducing infections caused by drug-resistant mutants in patients.

\section{Methods}

Viruses and cells. Wild-type HSV-1 strains (KOS, 294.1, and RE) and McKrae strain-derived LAT deletion mutant $d$ LAT2903 (kindly provided by G. C. Perng, Emory Vaccine Center, Emory University, Atlanta, Georgia, USA) were propagated and titrated on Vero cell monolayers. SK-N-SH cells (kindly provided by J.-I. Chuang, National Cheng Kung University) were infected with strain KOS at an MOI of 0.1.

RT-PCR analysis. Total RNA extracted from infected cells was reverse transcribed with an oligo (dT) or random hexamer primer. PCR amplification was performed on a thermal cycler, with denaturing for 1 minute at $94^{\circ} \mathrm{C}$, annealing for 1 minute at a temperature calculated from the primer set used, and extension for 1 minute at $72^{\circ} \mathrm{C}$ for a total of 30 cycles using primers for human Egr-1 (forward: $5^{\prime}$-CTGCACGCTTCTCAGTGTTC-3'; and reverse: 5'-AGCAGCATCATCTCCTCCAG-3') and $\beta$-actin (forward: $5^{\prime}$-ATCATGTTTGAGACCTTCAA-3'; and reverse: $5^{\prime}$ CATCTCTTGCTCGAAGTCCA-3').

Western blot analysis. Total proteins were extracted, blotted, and detected by using the antibodies against human Egr-1 (Santa Cruz Biotechnology Inc.), $\beta$-actin (Sigma-Aldrich), or $\alpha$-tubulin (Santa Cruz Biotechnology Inc.). The protein bands were developed by incubating with horseradish peroxidase-conjugated secondary antibodies (Santa Cruz Biotechnology Inc.) and with an enhanced chemiluminescence substrate kit (Millipore).
Infection of mice, tissue analyses, and DNAzyme treatment. All mouse experiment protocols were approved by the Laboratory Animal Committee at National Cheng Kung University. Egr $-1^{+/+}, E g r-1^{+/-}$, and Egr-1-1/- mice (mostly 6 to 8 weeks old) generated in a C57BL/6 background (kindly provided by J. Milbrandt, Washington University in St. Louis, St. Louis, Missouri, USA) were inoculated topically on the scarified cornea of the right eye with $1 \times 10^{7}$ PFUs of strain 294.1 or with $2 \times 10^{5}$ PFUs of strain $d$ LAT2903. The survival of infected mice was monitored. In separate experiments, mice were sacrificed and infected tissues were harvested for titration of infectious virus or for immunofluorescence staining. For immunofluorescence staining, tissue sections were stained with antibodies against Egr-1 and HSV-1 gD (8D2; kindly provided by R.N. Lausch, University of South Alabama, Mobile, Alabama, USA), and FITC- and rhodamine-conjugated secondary antibodies (Jackson ImmunoResearch Laboratories Inc.) were used to visualize these signals under a fluorescence microscope. For DNAzyme treatment, the scarified corneas of 5 -week-old C57BL/6 mice were treated with $5 \mu \mathrm{l}$ of solution containing $30 \mu \mathrm{g}$ of scramble oligmer (ED5SCR) or DNAzyme (ED5) against Egr-1 (33) (TriLink BioTechnologies), $\mathrm{MgCl}_{2}$ ( $25 \mathrm{mM}$ ), and $3 \mu \mathrm{l}$ of FuGENE 6 (Roche) 15 minutes before and from days 1 through 5 after being infected with $3 \times 10^{7} \mathrm{PFUs}$ of strain 294.1. In addition, the infected mice were given 1 intravenous injection of $200 \mu \mathrm{l}$ of solution containing $100 \mu \mathrm{g}$ of ED5SCR or ED5, $\mathrm{MgCl}_{2}(25 \mathrm{mM})$, and $3 \mu \mathrm{l}$ of FuGENE 6 daily from days 2 through 8 p.i.

Antisense repression. To knock down Egr-1 expression, SK-N-SH cells were transfected with a phosphorothioate antisense ( $5^{\prime}$-TsGsCsGsGsGsGsCGCGGGsGsAsAsCsAsCsT-3', where s indicates phosphorothioate moiety) oligodeoxynucleotide, which targets a sequence 119 to 100 bases upstream of the AUG initiation codon of human Egr-1, or a scrambled (5'-GsTsCsGsCsAsCsGCGTGCsGsAsGsGsAsGsG-3') oligodeoxynucleotide (25 $\mu \mathrm{M})$ as described $(20,21)$, using ESCORT Transfection Reagent (Sigma-Aldrich), and infected with strain KOS (MOI = 0.1) 6 hours later. Cultures were harvested to titrate for virus from 0 through 48 hours p.i.

Infection of mouse embryonic fibroblasts and brain slices. Embryonic fibroblasts were cultured from mice as described (40) and infected with strain KOS at an MOI of 0.01. Mouse brains were sliced with a tissue chopper into 1 - $\mathrm{mm}$ thickness. Brain slices $(0.06 \mathrm{~g}$ in weight) were infected in $2 \mathrm{ml}$ medium containing $8 \times 10^{5} \mathrm{PFUs}$ of strain KOS for 45 minutes. Infected brain slices were incubated in $1 \mathrm{ml}$ fresh medium for 24 hours, and the cultures were harvested and homogenized to titrate for virus.

Stable transfection. SK-N-SH cells were transfected with pCB6-Egr-1 (kindly provided by V. P. Sukhatme, Harvard Medical School, Boston, Massachusetts, USA), a vector expressing Egr-1, or vector pCB6 using lipofectin reagent (Invitrogen). Cells were expanded and selected in medium containing $400 \mu \mathrm{g} / \mathrm{ml}$ of G418 (Calbiochem) at 48 hours after transfection. Individual G418-resistant clones were isolated after drug selection for 3 to 4 weeks.

Construction of viral promoter reporter plasmids. To obtain HSV-1 promoters, primers for ICP4 (forward: 5'-GGAACGGAAGCGGAAACC-3'; and reverse: 5'-ACGGTCTGTCTCTGGCGGTC-3'), ICP27 (forward: 5'-TTGTCTGTGCCGGAGGTGG-3'; and reverse: 5'-AGCGTGACCGTGGTTGGAAC-3'), ICP22/47 (forward: 5'-GGTTTCCGCTTCCGTTCC-3'; and reverse: 5'CCTTTCCGATGCGATCCC-3'), and US6 (forward: $5^{\prime}$-TGTGACACTATCGTCCATACCG-3'; and reverse: 5'-TGATCGGGGTAGTGGTCGTTC-3') were used to amplify 361-, 148-, 554-, and 162-bp promoters, respectively, from strain RE. The PCR products were cloned into PCRII-TOPO vector (Invitrogen) and confirmed by sequencing. The plasmids containing the ICP4 or ICP27 promoter were digested with XhoI and HindIII; the plasmid containing the ICP22/47 promoter was digested with KpnI and XhoI; and the plasmid containing the US6 promoter was digested with XbaI and BamHI. The promoter fragments were cloned into pGL3 vector (Promega) to link 
with a luciferase reporter gene. To mutate the Egr-1-binding sequence in the ICP27 promoter from 5'-GCGGGGGCC-3' to 5'-TAAGCTTTA-3', a forward primer ( $5^{\prime}$-TTGTCTGTGCCGGAGGTGGGTAAGCTTTACCGC- $\left.{ }^{\prime}\right)$ and the ICP27 reverse primer were used to amplify a 148 -bp product from the ICP27 promoter plasmid. The PCR product was cloned into PCRII-TOPO vector and then into PGL3 reporter plasmid, as described for the ICP27 promoter.

Viral promoter reporter analysis. SK-N-SH cells stably transfected with pCB6-Egr-1 or pCB6 were transfected with $3 \mu \mathrm{g}$ of plasmids containing the viral promoters linked to a firefly luciferase reporter and $1 \mu \mathrm{g}$ of Renilla luciferase-expressing plasmid (pRL-TK; Promega) using lipofectin reagent (Invitrogen). Whole-cell lysates were extracted 48 hours after transfection, and luciferase activities were assayed using a Dual-Glo assay kit (Promega) and normalized to protein content. For each lysate, the firefly luciferase activity was normalized to the Renilla luciferase activity to assess transfection efficiencies.

ChIP-PCR assay. Clone 4 of SK-N-SH cells stably expressing Egr-1 was mock transfected or transfected with the ICP27 or mutated ICP27 promoter plasmid. At 48 hours after transfection, these transfectants were subjected to ChIP assay using the method previously described $(41,42)$, with modifications. In brief, the chromatins were immunoprecipitated by anti-Egr-1 antibody or rabbit IgG (Santa Cruz Biotechnology Inc.). After dissociating the DNA from proteins, the DNA was subjected to PCR amplification with denaturing for 1 minute at $94^{\circ} \mathrm{C}$, annealing for 1 minute and 30 seconds at $55^{\circ} \mathrm{C}$, and extension for 2 minutes at $72^{\circ} \mathrm{C}$ for a total of 30 cycles using the ICP27 forward and reverse primers to amplify 148-bp PCR products from the ICP27 and mutated ICP27 promoters.

Statistics. In vitro data were analyzed by Student's $t$ test. Viral loads in mouse tissues were analyzed by Mann-Whitney $U$ test. Kaplan-Meier sur- vival curves were analyzed by log-rank test. All $P$ values are for 2 -tailed significance tests. $P \leq 0.05$ was considered significant.

\section{Acknowledgments}

We thank D.M. Coen, R.N. Lausch, R. Anderson, and C.-C. Lee for critical reading; K.-S. Hsu, H.-Y. Lei, A.-L. Shiau, and S.-L Hung for helpful suggestions; and H.-M. Wu, C.-K. Yu, S.-J Tsai, K.-F Chen, P.-C. Chuang, and R.-H. Teng for technical help. This research was supported by grants from Taiwan National Science Council (95-2320-B-006-001 to Shun-Hua Chen and 95-2320-B-415-002 to $\mathrm{C}$. Li) and from Chung Shan Medical University (CSMU 92OM-B-016 to C. Li).

Received for publication January 23, 2008, and accepted in revised form July 16, 2008.

Address correspondence to: Shun-Hua Chen, Department of Microbiology and Immunology, College of Medicine, National Cheng Kung University, Tainan, Taiwan 701, Republic of China. Phone: 886-6-2353535 ext. 5633; Fax: 886-6-2082705; E-mail: shunhua@mail.ncku.edu.tw. Or to: Ching Li, Department of Microbiology and Immunology, College of Life Sciences, National Chiayi University, Chiayi, Taiwan 600, Republic of China. Phone: 886-5-2717833; Fax: 886-5-2717831; E-mail: chingli@mail.ncyu.edu.tw.

Shih-Heng Chen and Hui-Wen Yao contributed equally to this work.
1. Roizman, B., Knipe, D.M., and Whitley, R.J. 2007. Herpes simplex viruses. In Fields virology. D.M. Knipe and P. Howley, editors. Lippincott Williams \& Wilkins. Philadelphia, Pennsylvania, USA. 2501-2601.

2. Stanberry, L.R., Jorgensen, D.M., and Nahmias, A.J. 1997. Herpes simplex viruses 1 and 2. In Viral infections of humans: epidemiology and control. 4th edition. A.S. Evans and R.A. Kaslow, editors. Springer. New York, New York, USA. 419-454.

3. Zhang, S.Y., et al. 2007. TLR3 deficiency in patients with herpes simplex encephalitis. Science. 317:1522-1527.

4. Milbrandt, J. 1987. A nerve growth factor-induced gene encodes a possible transcriptional regulatory factor. Science. 238:797-799.

5. Lee, S.L., Tourtellotte, L.C., Wesselschmidt, R.L., and Milbrandt, J. 1995. Growth and differentiation proceeds normally in cells deficient in the immediate early gene NGFI-A. J. Biol. Chem. 270:9971-9977.

6. Thiel, G., and Cibelli, G. 2002. Regulation of life and death by the zinc finger transcription factor Egr-1. J. Cell Physiol. 193:287-292.

7. O'Donovan, K.J., Tourtellotte, W.G., Millbrandt, J., and Baraban, J.M. 1999. The EGR family of transcription-regulatory factors: progress at the interface of molecular and systems neuroscience. Trends Neurosci. 22:167-173.

8. Chauhan, D., et al. 1994. Involvement of serum response element in okadaic acid-induced EGR1 transcription in human T-cells. Cancer Res. 54:2234-2239.

9. Fu, Z.F., et al. 1993. Differential effects of rabies and borna disease viruses on immediate-earlyand late-response gene expression in brain tissues. J. Virol. 67:6674-6681.

10. Morimoto, K., et al. 1996. Intrinsic responses to Borna disease virus infection of the central nervous system. Proc. Natl. Acad. Sci. U. S. A. 93:13345-13350.

11. Sakamoto, K.M., Nimer, S.D., Rosenblatt, J.D., and Gasson, J.C. 1992. HTLV-I and HTLV-II tax trans-activate the human EGR-1 promoter through different cis-acting sequences. Oncogene. 7:2125-2130.

12. Wagner, A., et al. 2000. Induction of cellular genes is mediated by the Bel1 transactivator in foamy virus-infected human cells. J. Virol. 74:4441-4447.

13. Lee, S.L., et al. 1996. Luteinizing hormone deficiency and female infertility in mice lacking the transcription factor NGFI-A (Egr-1). Science. 273:1219-1221.

14. Chang, Y., et al. 2006. Induction of the early growth response 1 gene by Epstein-Barr virus lytic transactivator Zta. J. Virol. 80:7748-7755.

15. Zalani, S., Holley-Guthrie, E., and Kenney, S. 1995 The Zif268 cellular transcription factor activates expression of the Epstein-Barr virus immediateearly BRLF1 promoter. J. Virol. 69:3816-3823.

16. Nilsson, T., Zetterberg, H., Wang, Y.C., and Rymo, L. 2001. Promoter-proximal regulatory elements involved in oriP-EBNA1-independent and -dependent activation of the Epstein-Barr virus $\mathrm{C}$ promoter in B-lymphoid cell lines. J. Virol. 75:5796-5811.

17. Tatarowicz, W.A., et al. 1997. Repression of the HSV-1 latency-associated transcript (LAT) promoter by the early growth response (EGR) proteins: involvement of a binding site immediately downstream of the TATA box. J. Neurovirol. 3:212-224.

18. Sacks, S.L., et al. 1989. Progressive esophagitis from acyclovir-resistant herpes simplex. Clinical roles for DNA polymerase mutants and viral heterogeneity? Ann. Intern. Med. 111:893-899.

19. Chen, S.H., et al. 2006. Efficient reactivation of latent herpes simplex virus from mouse central nervous system tissues. J. Virol. 80:12387-12392.

20. Muthukkumar, S., et al. 1995. Role of EGR-1 in thapsigargin-inducible apoptosis in the melanoma cell line A375-C6. Mol. Cell. Biol. 15:6262-6272.

21. Nguyen, H.Q., Hoffman-Liebermann, B., and Liebermann, D.A. 1993. The zinc finger transcription factor Egr-1 is essential for and restricts differentiation along the macrophage lineage. Cell. 72:197-209.
22. Perng, G.C., et al. 2001. Three herpes simplex virus type 1 latency-associated transcript mutants with distinct and asymmetric effects on virulence in mice compared with rabbits. J. Virol. 75:9018-9028.

23. Leib, D.A., et al. 1989. A deletion mutant of the latency-associated transcript of herpes simplex virus type 1 reactivates from the latent state with reduced frequency. J. Virol. 63:2893-2900.

24. Hosono, S., et al. 2000. E-cadherin is a WT1 target gene. J. Biol. Chem. 275:10943-10953.

25. Nishi, H., Nishi, K.H., and Johnson, A.C. 2002. Early Growth Response-1 gene mediates up-regulation of epidermal growth factor receptor expression during hypoxia. Cancer Res. 62:827-834.

26. Christy, B., and Nathans, D. 1989. DNA binding site of the growth factor-inducible protein Zif268. Proc. Natl. Acad. Sci. U. S. A. 86:8737-8741.

27. Ebert, S.N., and Wong, D.L. 1995. Differential activation of the rat phenylethanolamine N-methyltransferase gene by Sp1 and Egr-1. J. Biol. Chem. 270:17299-17305.

28. Virolle, T., et al. 2001. The Egr-1 transcription factor directly activates PTEN during irradiationinduced signalling. Nat. Cell Biol. 3:1124-1128.

29. Ralph, W.M., Jr., Cabatingan, M.S., and Schaffer, P.A. 1994. Induction of herpes simplex virus type 1 immediate-early gene expression by a cellular activity expressed in Vero and NB41A3 cells after growth arrest-release. J. Virol. 68:6871-6882.

30. Gelman, I.H., and Silverstein, S. 1987. Herpes simplex virus immediate-early promoters are responsive to virus and cell trans-acting factors. J. Virol. 61:2286-2296

31. Sze, P., and Herman, R.C. 1992. The herpes simplex virus type 1 ICP 6 gene is regulated by a 'leaky' early promoter. Virus Res. 26:141-152.

32. Huang, C.J., and Wagner, E.K. 1994. The herpes simplex virus type 1 major capsid protein (VP5UL19) promoter contains two cis-acting elements influencing late expression. J. Virol. 68:5738-5747.

33. Fahmy, R.G., Dass, C.R., Sun, L.Q., Chesterman, 
C.N., and Khachigian, L.M. 2003. Transcription factor Egr-1 supports FGF-dependent angiogenesis during neovascularization and tumor growth. Nat. Med. 9:1026-1032.

34. Santiago, F.S., et al. 1999. New DNA enzyme targeting Egr-1 mRNA inhibits vascular smooth muscle proliferation and regrowth after injury. Nat. Med. 5:1264-1269.

35. Aicher, W.K., Sakamoto, K.M., Hack, A., and Eibel, H. 1999. Analysis of functional elements in the human Egr-1 gene promoter. Rheumatol. Int. 18:207-214.

36. Bittlingmaier, K., Schneider, D., and Falke, D. 1977. Influence of dibutyryl cyclic AMP on thymidine uptake by herpes simplex virus infected cells and the intracellular level of cyclic AMP. Biochim. Biophys. Acta. 477:228-238.

37. Kim, D.B., and DeLuca, N.A. 2002. Phosphorylation of transcription factor Sp1 during herpes simplex virus type 1 infection. J. Virol. 76:6473-6479.

38. Jang, K.L., Pulverer, B., Woodgett, J.R., and Latchman, D.S. 1991. Activation of the cellular transcription factor AP-1 in herpes simplex virus infected cells is dependent on the viral immediate-early protein ICPO. Nucleic Acids Res. 19:4879-4883.

39. Casrouge, A., et al. 2006. Herpes simplex virus encephalitis in human UNC-93B deficiency. Science. 314:308-312.
40. Smith, C.L. 2007. Mouse embryo fibroblast (MEF) feeder cell preparation. In Current protocols in molecular biology. F.M. Ausubel, et al., editors. John Wiley \& Sons. New York, New York, USA. 28.21.21-28.21.23.

41. Sun, H.S., Hsiao, K.Y., Hsu, C.C., Wu, M.H., and Tsai, S.J. 2003. Transactivation of steroidogenic acute regulatory protein in human endometriotic stromalcells is mediated by the prostaglandin EP2 receptor. Endocrinology. 144:3934-3942.

42. Chen, K.F., Lai, Y.Y., Sun, H.S., and Tsai, S.J. 2005. Transcriptional repression of human cad gene by hypoxia inducible factor-1alpha. Nucleic Acids Res. 33:5190-5198. 